Proceedings of the Institute of Mathematics and Mechanics,

National Academy of Sciences of Azerbaijan

Volume 46, Number 2, 2020, Pages 284-293

https://doi.org/10.29228/proc.33

\title{
DISCRETE MAXIMUM PRINCIPLE IN SYSTEMS WITH A DELAY IN CONTROL
}

\author{
MISIR J. MARDANOV AND SAMIN T. MALIK
}

\begin{abstract}
In the paper, we consider a discrete optimal control problem with a delay in control. Taking into account the specificity of this problem, we offer a new research method. The essence of this method is that unlike previously known ones here we assume that the set of admissible speeds satisfies convexity type conditions at the moment $t+h$, but not at $t$, where $h$ is time delay in the system. The discrete analog of Pontryagin's maximum principle is obtained under weakened assumptions. An example showing the content-richness of the obtained result is given.
\end{abstract}

\section{Introduction}

As known, research of optimization problems of continuous and discrete systems with a delay was developed after the proof of Pontryagin's maximum principle [27]. As a result, in numerous works [3,5,7,9,13,16,17,23-26 and others] as a rule, the analogs of Pontryagin's maximum principle were obtained.

After A.G.Butkovsky's work [2] it became known that generally speaking the analog of Pontryagin's maximum principle does not hold for a nonlinear discrete optimization problem. Therefore, we can accept that among optimal control problems the discrete optimal control problems (even without a delay) occupy a special place and, naturally, are of theoretical interest. Furthermore, discrete optimization problems are also of practical interest since mathematical modeling of economic planning, organization of production and some other processes that can be modeled by optimization problems with a delay in control (see for example [4],[10]). History of occurrence and development of discrete optimal control problems (with and without delay) was stated, for example, in $[1,6,14,15,20$, $21,26]$.

Analyzing works $[9,11,14,19,23,29]$, we can note that when studying optimal control problems with a delay in control, special difficulties arise. The cause is that the methods offered and worked out for obtained discrete analogue of Pontryagin's maximum principle, for example in $[8,28]$, directly can not be applicable or can be applied under strict assumptions. More exactly, as far as we know, the discrete analog of Pontryagin's maximum principle for the optimization problem with a delay in control was obtained only in the paper [29] but under rather

2010 Mathematics Subject Classification. 49K15, 34H05.

Key words and phrases. Discrete maximum principle, delay control systems, optimal control. 
strict assumptions. Generally speaking, the method offered in [29], can not be used even for linear optimization problems with a delay in control. We should note that in [12], giving a specific example, it is shown that discrete analog of the maximum principle for discrete optimal control problems with a delay in control that are linear only by their state, does not always hold. As seen, these circumstances require a new approach to the study of a discrete optimization problem with a delay in control. It is natural that realization of such a requirement is still of current interest.

This paper was devoted to the study of discrete optimization problem with a delay in control for the case when the set of values of admissible controls is arbitrary. Taking into account the specifics of considered system, we offer a new research method. Herewith, unlike the earlier known ones (see $[8,12,22,28]$ ), we assume that the set of admissible speeds satisfies the convexity type condition at the moment $t+h$, but not at $t$, where $h$ is time delay in the system. As a result, we get a discrete analog of Pontryagin's maximum principle. An example showing content-richness of the obtained result is given.

\section{Problem statement}

We consider the following discrete optimization problem with a delay in control:

$$
\begin{gathered}
S(u(\cdot))=\Phi\left(x\left(t_{1}\right)\right) \rightarrow \min _{u(\cdot)}, \\
x(t+1)=f(x(t), u(t), u(t-h), t), \\
t \in I:=\left\{t_{0}, t_{0}+1 \ldots, t_{1}-1\right\}, x\left(t_{0}\right)=x_{0}, \\
u(t) \in U(t) \subseteq R^{r}, t \in\left\{t_{0}-h, \ldots, t_{0}, \ldots, t_{1}-1\right\}=: I_{h},
\end{gathered}
$$

where $R^{r}$ is $r$-dimensional Euclidean space, $x(\cdot) \in R^{n}$ is a state vector, $u(\cdot) \in R^{r}$ is a control vector, $t$ is time (discrete), $t_{0}, t_{1} \in R, h \in N=\{1,2, \ldots\}, x_{0} \in R^{n}$ are the given points, moreover $t_{1}-t_{0}>h, \Phi(x): R^{n} \rightarrow R$ and $f(x, u, w, t)$ : $R^{n} \times R^{r} \times R^{r} \times I \rightarrow R^{n}$ are the given functions, $U(t), t \in I_{h}$ are the given sets.

We call the control $u(t), t \in I_{h}$, satisfying (2.3) an admissible control, appropriate solution $x(t), t \in I \bigcup\left\{t_{1}\right\}$ of the system (2.2) an admissible trajectory. We call the pair $(u(\cdot), x(\cdot))$ an admissible process. The admissible control $u(t), t \in I_{h}$, that affords minimum to the functional (2.1) under the constraint (2.2) is called optimal control, the appropriate solution $x(t), t \in I \bigcup\left\{t_{1}\right\}$ of the system $(2.2)$ an optimal trajectory. We call the pair $(u(\cdot), x(\cdot))$ an optimal process.

As seen, the system (2.2) is not the most general of the discrete systems with a delay in control. It was chosen by us only to simplify the formulation and the proof of the main result, and also to state the essence of the offered approach.

For studying problem $(2.1)-(2.3)$ we assume that the functional $\Phi(\cdot)$ is continuously differentiable and furthermore, for each $t \in I$ the function $f(\cdot, \cdot, \cdot, t)$ and its partial derivative $f_{x}(\cdot, \cdot, \cdot, t)$ are continuous with respect to $(x, u, w)$.

When studying problem (2.1)- (2.3) we will use the following definition.

Definition 2.1. [18] The set $Z \subset E^{m}$ is said to be $\gamma$ - convex with respect to the point $z_{0} \in Z$ if for each point $z \in Z$ there exists such a point $\gamma=\gamma(z) \in(0,1]$ that for all $\varepsilon \in(0, \gamma]$ the inclusion $z_{0}+\varepsilon\left(z-z_{0}\right) \in Z$ is valid. If the set $Z$ is $\gamma$ convex with respect to each of its points, we call it $\gamma$ - convex. 


\section{Formulas for the increment of the quality functional}

The proof of the maximum principle for problem (2.1)- (2.3) is based on the result obtained in this section. It should be noted that this result has an independent meaning.

Let $(\bar{u}(\cdot), \bar{x}(\cdot))$ be some admissible process and $(\theta, \vartheta) \in I_{h} \times U(\theta)$ be an arbitrary fixed point. Considering the following three cases, we find formulas for the increment of the functional (2.1):

Case 3.1. Let $\theta \in\left\{t_{0}-h, t_{0}-h+1, \ldots, t_{0}-1\right\}$ and suppose that the set $f(\bar{x}(\theta+h), \bar{u}(\theta+h), U(\theta), \theta+h)$ is $\gamma$ - convex with respect to the point $\bar{x}(\theta+h+1)$ (in the sense of definition 2.1).

We determine the variation of the control $\bar{u}(t), t \in I_{h}$ as follows:

$$
u(t ; \theta, \vartheta, \varepsilon)=\left\{\begin{array}{l}
\vartheta(\varepsilon), \quad t=\theta, \\
\bar{u}(t), \quad t \in I_{h} \backslash\{\theta\} .
\end{array}\right.
$$

Here the function is determined $\vartheta(\varepsilon):(0, \gamma] \rightarrow U(\theta)$ is defined (in the implicit form) as the solution of the system

$$
\begin{gathered}
\bar{f}(\bar{x}(\theta+h), \bar{u}(\theta+h), \vartheta(\varepsilon), \theta+h)-\bar{f}(\theta+h)= \\
=\varepsilon \Delta_{(\bar{u}(\theta+h), \vartheta)} \bar{f}(\theta+h), \varepsilon \in(0, \gamma],
\end{gathered}
$$

where $(0, \gamma] \subset[0,1]$, the number $\gamma=\gamma(\vartheta)$ is given according to definition 2.1,

$$
\begin{gathered}
\bar{f}(\tau)=f(\bar{x}(\tau), \bar{u}(\tau), \bar{u}(\tau-h), \tau), \\
\Delta_{(\bar{u}(\tau), \vartheta)} \bar{f}(\tau):=f(\bar{x}(\tau), \bar{u}(\tau), \vartheta, \tau)-\bar{f}(\tau), \tau=\theta+h, \vartheta \in U(\theta) .
\end{gathered}
$$

By definition 2.1, such a solution exists since the set $f(\bar{x}(\tau), \bar{u}(\tau), U(\theta), \tau)$ is $\gamma$ - convex with respect to the point $\bar{f}(\tau)$, where $\tau=\theta+h$.

Obviously, for all $\varepsilon \in(0, \gamma]$ the function $u(\cdot, \theta, \vartheta, \varepsilon)$ is an admissible control.

Let us consider an admissible process $(u(\cdot ; \theta, \vartheta, \varepsilon), x(\cdot ; \theta, \vartheta, \varepsilon))$. It is clear that the increment $x(t ; \theta, \vartheta, \varepsilon)-\bar{x}(t)=: \Delta x(t ; \theta, \vartheta, \varepsilon), t \in I \cup\left\{t_{1}\right\}$ is the solution of the system

$$
\left\{\begin{aligned}
& \Delta x(t+1 ; \theta, \vartheta, \varepsilon)= f(\bar{x}(t)+\Delta x(t ; \theta, \vartheta, \varepsilon), u(t ; \theta, \vartheta, \varepsilon), \\
&u(t-h ; \theta, \vartheta, \varepsilon), t)-\bar{f}(t), \\
& \Delta x\left(t_{0} ; \theta, \vartheta, \varepsilon\right)=0, \varepsilon \in(0, \gamma] .
\end{aligned}\right.
$$

Taking into account (3.1)-(3.3), we can write the system (3.4) in the form:

$$
\Delta x \in(t+1 ; \theta, \vartheta, \varepsilon)= \begin{cases}0, & t_{0}-1 \leq t<\theta+h, \\ \varepsilon \Delta_{(\bar{u}(\theta+h), \vartheta)} \bar{f}(\theta+h), & t=\theta+h, \\ \Delta_{x(t ; \theta, \vartheta, \varepsilon)} f(t), & \theta+h<t<t_{1} .\end{cases}
$$

Here $\varepsilon \in(0, \gamma]$, while $\Delta_{(\bar{u}(\theta+h), \vartheta)} \bar{f}(\theta+h)$ is determined by (3.3),

$$
\begin{aligned}
\Delta_{x(t ; \theta, \vartheta, \varepsilon)} \bar{f}(t)= & f(x(t ; \theta, \vartheta, \varepsilon), \bar{u}(t), \bar{u}(t-h), t)-\bar{f}(t), \\
& t \in\left\{\theta+h+1, \ldots, t_{1}-1\right\},
\end{aligned}
$$

where $x(t ; \theta, \vartheta, \varepsilon)=\bar{x}(t)+\Delta x(t ; \theta, \vartheta, \varepsilon)$.

Using the Taylor formula, from (3.5), allowing for (3.6) and applying the method of steps, it is easy to obtain

$$
\|\Delta x(t ; \theta, \vartheta, \varepsilon)\| \leq K \varepsilon, t \in I \cup\left\{t_{1}\right\}, \varepsilon \in(0, \gamma], K=\text { const }>0,
$$


where $\|\cdot\|$ is Euclidean norm.

Now, taking into account (3.1) and (3.5)-(3.7), we calculate the increment

$$
S(u(\cdot ; \theta, \vartheta, \varepsilon))-S(\bar{u}(\cdot))=: \Delta_{(\theta, \vartheta, \varepsilon)} S(\bar{u}(\cdot))
$$

of first order of the functional (2.1).

We consider the auxiliary vector-function $\bar{\psi}(t), t \in I$ corresponding to the process $(\bar{u}(\cdot), \bar{x}(\cdot))$ as the solution of the system in the form

$$
\begin{gathered}
\bar{\psi}(t-1)=\bar{f}_{x}^{T}(t) \bar{\psi}(t), t \in\left\{t_{0}+1, t_{0}+2, \ldots, t_{1}-1\right\}, \\
\bar{\psi}\left(t_{1}-1\right)=-\Phi_{x}\left(\bar{x}\left(t_{1}\right)\right) .
\end{gathered}
$$

By the Taylor formula, allowing for (3.7), we have

$$
\Delta_{(\theta, u, \varepsilon)} S(\bar{u}(\cdot))=\Phi_{x}^{T}\left(\bar{x}\left(t_{1}\right)\right) \Delta x\left(t_{1} ; \theta, \vartheta, \varepsilon\right)+o(\varepsilon), \varepsilon \in(0, \gamma],
$$

where $o(\varepsilon) / \varepsilon \rightarrow 0$, as $\varepsilon \rightarrow+0$.

Using (3.5) and (3.8), we can write equality (3.9) in the following form:

$$
\begin{aligned}
& \Delta_{(\theta, \vartheta, \varepsilon)} S(\bar{u}(\cdot))=\bar{\psi}^{T}\left(t_{1}-1\right) \Delta x\left(t_{1} ; \theta, \vartheta, \varepsilon\right)+o(\varepsilon)= \\
= & -\varepsilon \bar{\psi}^{T}(\tau) \Delta_{(\bar{u}(\tau), \vartheta)} \bar{f}(\tau)-\sum_{t=\tau+1}^{t_{1}-1} \bar{\psi}^{T}(t) \Delta_{x(t ; \theta, \vartheta, \varepsilon)} \bar{f}(t)+ \\
& +\sum_{t=\tau+1}^{t_{1}-1} \bar{\psi}^{T}(t-1) \Delta x(t ; \theta, \vartheta, \varepsilon)+o(\varepsilon), \quad \varepsilon \in(0, \gamma]
\end{aligned}
$$

where $\tau=\theta+h$.

Hence, taking into account (3.6)-(3.8), by the Taylor formula we get the soughtfor formula for case 3.1:

$$
\Delta_{(\theta, \vartheta, \varepsilon)} S(\bar{u}(\cdot))=-\varepsilon \bar{\psi}^{T}(\theta+h) \Delta_{(\bar{u}(\theta+h), \vartheta)} \bar{f}(\theta+h)+o(\varepsilon), \varepsilon \in(0, \gamma],
$$

where $\Delta_{(\bar{u}(\theta+h), \vartheta)} \bar{f}(\theta+h)$ is determined by (3.3).

Case 3.2. Let $\theta \in\left\{t_{0}, t_{0}+1, \ldots, t_{1}-h-1\right\}$ and the set $f\left(f^{[h-1]}(\theta+h-1\right.$, $U(\theta)) \bar{u}(\theta+h), U(\theta), \theta+h)$ be $\gamma$ - convex with respect to the point $\bar{x}(\theta+h+1)$, where the function $f^{[h-1]}(\theta+h-1, \vartheta)$ with respect to the variable $\vartheta \in U(\theta)$ is determined as follows:

$$
\left\{\begin{array}{l}
f^{[k]}(\theta+k, \vartheta)=f\left(f^{[k-1]}(\theta+k-1, \vartheta), \bar{u}(\theta+k), \bar{u}(\theta+k-h), \theta+k\right), \\
f^{[0]}(\theta, \vartheta)=f(\bar{x}(\theta), \vartheta, \bar{u}(\theta-h), \theta), k=1,2, \ldots, h .
\end{array}\right.
$$

Obviously, $\bar{x}(\theta+h+1)=f\left(f^{[h-1]}(\theta+h-1, \bar{u}(\theta)), \bar{u}(\theta+h), \bar{u}(\theta), \theta+h\right)$.

The following equality is also valid

$$
f^{[k-1]}(\theta+k-1, \bar{u}(\theta))=\bar{x}(\theta+k), \text { for all } k \in\{1,2, \ldots, h\} .
$$

Let us consider variation (3.1), moreover $\theta \in\left\{t_{0}, t_{0}+1, \ldots, t_{1}-h-1\right\}$, i.e. $t_{0} \leq \theta<t_{1}-h$, and the function $\vartheta(\varepsilon):[0, \gamma] \rightarrow U(\theta)$ is determined (in the implicit form) as the solution of the system

$$
\begin{aligned}
& f\left(f^{[h-1]}(\tau-1, \vartheta(\varepsilon)), \bar{u}(\tau), \vartheta(\varepsilon), \tau\right)-f\left(f^{[h-1]}(\tau-1, \bar{u}(\theta)), \bar{u}(\tau), \bar{u}(\theta), \tau\right)= \\
& =\varepsilon\left[f\left(f^{[h-1]}(\tau-1, \vartheta), \bar{u}(\tau), \vartheta, \tau\right)-f\left(f^{[h-1]}(\tau-1, \bar{u}(\theta)), \bar{u}(\tau), \bar{u}(\theta), \tau\right)\right],
\end{aligned}
$$




$$
\varepsilon \in(0, \gamma]
$$

where $\tau=\theta+h,(0, \gamma] \subset(0,1]$, the number $\gamma=\gamma(\vartheta)$ is given by definition 2.1, while $f^{[h-1]}(\tau-1, \vartheta(\varepsilon))$ and $f^{[h-1]}(\tau-1, \vartheta)$ are determined by $(3.11)$.

Note that since the set $f\left(f^{[h-1]}(\theta+h-1, U(\theta)), \bar{u}(\theta+h), U(\theta), \theta+h\right)$ is $\gamma$ - convex with respect to the point $\bar{x}(\theta+h+1)$, i.e. to the point $f\left(f^{[h-1]}(\theta+h-1, \bar{u}(\theta)), \bar{u}(\theta+h)\right.$, $\bar{u}(\theta), \theta+h)$, then by definition 2.1 the solution $\vartheta(\varepsilon), \varepsilon \in(0, \gamma]$ exists.

In this case, considering (3.1), (3.12)-(3.15), we can write the system (3.4) in the following form:

$$
\begin{gathered}
\Delta x(t+1 ; \theta, \vartheta, \varepsilon)= \\
=\left\{\begin{array}{lc}
0, & t_{0}-1 \leq t<\theta, \\
f^{[t-\theta]}(t, \vartheta(\varepsilon))-\bar{f}(t), & \theta \leq t<\theta+h, \\
\varepsilon\left[f\left(f^{[h-1]}(\theta+h-1, \vartheta), \bar{u}(\theta+h), \vartheta, \theta+h\right)-\bar{f}(\theta+h)\right], & t=\theta+h, \\
\Delta_{x(t ; \theta, \vartheta, \varepsilon)} \bar{f}(t), & \theta+h<t<t_{1} .
\end{array}\right.
\end{gathered}
$$

By the Taylor formula, from (3.14) allowing for (3.6) and by means of the method of steps, we get

$$
\|\Delta x(t ; \theta, \vartheta, \varepsilon)\| \leq K \varepsilon, t \in\left\{\theta+h+1, \ldots, t_{1}\right\}, \varepsilon \in(0, \gamma], K=\text { const }>0 .
$$

We consider the following identity :

$$
\begin{aligned}
\bar{\psi}^{T}\left(t_{1}-1\right) \Delta & x\left(t_{1} ; \theta, \vartheta, \varepsilon\right)=\sum_{t=\theta+h}^{t_{1}-1} \bar{\psi}^{T}(t) \Delta x(t+1 ; \theta, \vartheta, \varepsilon)- \\
- & \sum_{t=\theta+h+1}^{t_{1}-1} \bar{\psi}^{T}(t-1) \Delta x(t ; \theta, \vartheta, \varepsilon),
\end{aligned}
$$

where the functions $\bar{\psi}(\cdot)$ and $\Delta x(\cdot ; \theta, \vartheta, \varepsilon), \varepsilon \in(0, \gamma]$ are determined by (3.8) and (3.14), respectively. At first we take into account (3.14) in (3.16), and then taking into account (3.6) and (3.15), we apply the Taylor formula. As a result, by (3.8) we get

$$
\begin{gathered}
\bar{\psi}^{T}\left(t_{1}-1\right) \Delta x\left(t_{1} ; \theta, \vartheta, \varepsilon\right)=\varepsilon \bar{\psi}^{T}(\theta+h) \times \\
\times\left[f\left(f^{[h-1]}(\theta+h-1, \vartheta), \bar{u}(\theta+h), \vartheta, \theta+h\right)-\bar{f}(\theta+h)\right]+o(\varepsilon) .
\end{gathered}
$$

Since $\bar{\psi}(t-1)=-\Phi_{x}\left(\bar{x}\left(t_{1}\right)\right)$, then in this case, allowing for (3.17) the increment (3.9) takes the form

$$
\begin{array}{r}
\Delta_{(\theta, \vartheta, \varepsilon)} S(\bar{u}(\cdot))=-\varepsilon \bar{\psi}^{T}(\theta+h)\left[f \left(f^{[h-1]}(\theta+h-1, \vartheta),\right.\right. \\
\bar{u}(\theta+h), \vartheta, \theta+h)-\bar{f}(\theta+h)]+o(\varepsilon), \vartheta \in U(\theta), \varepsilon \in(0, \gamma],
\end{array}
$$

where $f^{[h-1]}(\theta+h-1, \vartheta)$ is determined by (3.11).

Case 3.3. Let $\theta \in\left\{t_{1}-h, t_{1}-h+1, \ldots, t_{1}-1\right\}$ and let the set $f(\bar{x}(\theta), U(\theta)$, $\bar{u}(\theta-h), \theta)$ be $\gamma$ - convex with respect to the point $\bar{x}(\theta+1)$.

In this case, obtaining the increment formula of the quality functional (2.1) is carried out quite similarly to case 1 . More exactly, here as a variation of the 
control $\bar{u}(t), t \in I_{h}$ we choose $(3.1)$, wherein now the function $\vartheta(\varepsilon):(0, \gamma] \rightarrow$ $U(\theta)$ is determined (in the implicit form) as the solution of the system

$$
\begin{gathered}
f(\bar{x}(\theta), \vartheta(\varepsilon), \bar{u}(\theta-h), \theta)-\bar{f}(\theta)= \\
=\varepsilon(f(\bar{x}(\theta), \vartheta, \bar{u}(\theta-h), \theta)-\bar{f}(\theta)), \varepsilon \in(0, \gamma],
\end{gathered}
$$

where $(\theta, \gamma] \subset(\theta, 1]$, the number $\gamma=\gamma(\vartheta)$ is given according to definition 2.1.

In what follows, similar to (3.5), taking into account (3.19), we can write the system (3.4) in the following form:

$$
\Delta x(t+1 ; \theta, \vartheta, \varepsilon)= \begin{cases}0, & t_{0}-1 \leq t<\theta, \\ \varepsilon[f(\bar{x}(\theta), \vartheta, \bar{u}(\theta-h), \theta)-\bar{f}(\theta)], & t=\theta, \\ \Delta_{x(t ; \theta, \vartheta, \varepsilon)} \bar{f}(t), & \theta<t<t_{1},\end{cases}
$$

where $\varepsilon \in(0, \gamma], \Delta_{x(t ; \theta, \vartheta, \varepsilon)} \bar{f}(t)$ is determined by (3.6).

From (3.20), similar to (3.7), we have

$$
\|\Delta x(t ; \theta, \vartheta, \varepsilon)\| \leq K \varepsilon, t \in I \cup\left\{t_{1}\right\}, \varepsilon \in(0, \gamma], K=\text { const }>0 .
$$

Using an identity of the form

$$
\begin{gathered}
\bar{\psi}^{T}\left(t_{1}-1\right) \Delta x\left(t_{1} ; \theta, \vartheta, \varepsilon\right)=\bar{\psi}^{T}(\theta) \Delta x(\theta+1 ; \theta, \vartheta, \varepsilon)+ \\
+\sum_{t=\theta+1}^{t_{1}-1} \bar{\psi}^{T}(t) \Delta x(t+1 ; \theta, \vartheta, \varepsilon)-\sum_{t=\theta+1}^{t_{1}-1} \bar{\psi}^{T}(t-1) \Delta x(t, \theta, \vartheta, \varepsilon)
\end{gathered}
$$

and taking into account (3.8), (3.20), by the Taylor formula for the increment (3.9) we get the following sought-for formula:

$$
\begin{gathered}
\Delta_{(\theta, \vartheta, \varepsilon)} S(\bar{u}(\cdot))=-\varepsilon \bar{\psi}^{T}(\theta)[f(\bar{x}(\theta), \vartheta, \bar{u}(\theta-h), \theta)-\bar{f}(\theta)]+o(\varepsilon) \\
\vartheta \in U(\theta), \varepsilon \in(0, \gamma] .
\end{gathered}
$$

Thus, we prove the following statement.

Proposition 3.1. Let the function $\Phi(\cdot)$ be continuously differentiable, and furthermore for each $t \in I$ the function $f(\cdot, \cdot, \cdot, t)$ and its partial derivative $f_{x}(\cdot, \cdot, \cdot, t)$ be continuous with respect to $(x, u, w)$. Further, let $(\bar{u}(\cdot), \bar{x}(\cdot))$ be some admissible process. Then:

(1) if for the point $\theta \in\left\{t_{0}-h, t_{0}-h+1, \ldots, t_{0}-1\right\}$ the set $f(\bar{x}(\theta+h), \bar{u}(\theta+h), U(\theta), \theta+h)$ is $\gamma$ - convex with respect to the point $\bar{x}(\theta+h+1)$, then formula (3.10) is valid;

(2) if for the point $\theta \in\left\{t_{0}, t_{0}+1, \ldots, t_{1}-h-1\right\}$ the set $f\left(f^{[h-1]}(\theta+h-1, U(\theta)), \bar{u}(\theta+h), U(\theta), \theta+h\right)$ is $\gamma$ - convex with respect to the point $\bar{x}(\theta+h+1)$, then formula (3.18) holds, where the function $f^{[h-1]}(\theta+h-1, \vartheta)$ is determined by (3.11);

(3) if for the point $\theta \in\left\{t_{1}-h, t_{1}-h+1, \ldots, t_{1}-1\right\}$ the set $f(\bar{x}(\theta), U(\theta), \bar{u}(\theta-h), \theta)$ is $\gamma$ - convex with respect to the point $\bar{x}(\theta+1)$, then formula $(3.22)$ is valid. 


\section{Optimality conditions}

Based on proposition 3.1, we prove the following theorem.

Theorem 4.1. Let the functional $\Phi(\cdot)$ be continuously differentiable, and furthermore for each $t \in I$ the function $f(\cdot, \cdot, \cdot, t)$ and its partial derivative $f_{x}(\cdot, \cdot, \cdot, t)$ be continuous with respect to $(x, u, w)$. Further, let the admissible process $(\bar{u}(\cdot), \bar{x}(\cdot))$ be optimal and the function $\bar{\psi}(\cdot), t \in I$ be the solution of the system (3.8). Then:

(1) if for each $\theta \in\left\{t_{0}-h, t_{0}-h+1, \ldots, t_{0}-1\right\}$ the set $f(\bar{x}(\theta+h), \bar{u}(\theta+h)$, $U(\theta), \theta+h)$ is $\gamma$-convex with respect to the point $\bar{x}(\theta+h+1)$, then for all $\vartheta \in U(\theta)$ we have the inequality

$$
\bar{\psi}^{T}(\theta+h)[f(\bar{x}(\theta+h), \bar{u}(\theta+h), \vartheta, \theta+h)-\bar{x}(\theta+h+1)] \leq 0 ;
$$

(2) if for each $\theta \in\left\{t_{0}, t_{0}+1, \ldots, t_{1}-h-1\right\}$ the set

$f\left(f^{[h-1]}(\theta+h-1, U(\theta)), \bar{u}(\theta+h), U(\theta), \theta+h\right)$ is $\gamma$ - convex with respect to the point $\bar{x}(\theta+h+1)$, then for all $\vartheta \in U(\theta)$ the following inequality is valid

$$
\bar{\psi}^{T}(\theta+h)\left[f\left(f^{[h-1]}(\theta+h-1, \vartheta), \bar{u}(\theta+h), \vartheta, \theta+h\right)-\bar{x}(\theta+h+1)\right] \leq 0,
$$

where $f^{[h-1]}(\theta+h-1, \vartheta)$ is determined by (3.11);

(iii) if for each $\theta \in\left\{t_{1}-h, t_{1}-h+1, \ldots, t_{1}-1\right\}$ the set $f(\bar{x}(\theta), U(\theta)$, $\bar{u}(\theta-h), \theta)$ is $\gamma$-convex with respect to the point $\bar{x}(\theta+1)$, then for all $\vartheta \in U(\theta)$ the following inequality is valid

$$
\bar{\psi}^{T}(\theta)[f(\bar{x}(\theta), \vartheta, u(\theta-h), \theta)-\bar{x}(\theta+1)] \leq 0 .
$$

Proof. At first we prove part (i) of theorem 4.1. Since for each $\theta \in\left\{t_{0}-h, t_{0}-h+1, \ldots, t_{0}-1\right\}$ the set $f(\bar{x}(\theta+h), \bar{u}(\theta+h), U(\theta), \theta+h)$ is $\gamma$-convex with respect to the point $\bar{x}(\theta+h+1)$, then by (i) part of Proposition 3.1 , allowing for $(3.3)$ and $\bar{f}(\theta+h)=\bar{x}(\theta+h+1)$ along the process $(\bar{u}(\cdot), \bar{x}(\cdot))$, for arbitrary fixed $\vartheta \in U(\theta)$ the following equality holds

$$
\begin{aligned}
\Delta_{(\theta, \vartheta, \varepsilon)} S(\bar{u}(\cdot)) & =-\varepsilon \bar{\psi}^{T}(\theta+h)[f(\bar{x}(\theta+h), \bar{u}(\theta-h), \vartheta, \theta+h)- \\
& -\bar{x}(\theta+h+1)]+o(\varepsilon), \varepsilon \in(0, \gamma] .
\end{aligned}
$$

Since the process $(\bar{u}(\cdot), \bar{x}(\cdot))$ is optimal, then $\Delta_{(\theta, \vartheta, \varepsilon)} S(\bar{u}(\cdot)) \geq 0$ for all $\varepsilon \in(0, \gamma]$. Therefore, having divided the obtained expression for $\Delta_{(\theta, \vartheta, \varepsilon)} S(\bar{u}(\cdot))$ into $\varepsilon$ and passing to limit as $\varepsilon \rightarrow+0$, we get the sought-for inequality (4.1). Thus, part (i) of theorem 4.1 is proved.

Quite similarly, using (ii) and (iii) parts of Proposition 3.1, we prove parts (ii) and (iii) of theorem 4.1. Theorem 4.1 is proved.

Remark 4.1. If in the problem (2.1)-(2.3)

$$
f(\cdot)=A(u, t) x+b(u, w, t) \text { and } \Phi(x)=c^{T} x,
$$

where $A(u, t)$ is $n \times n$ - matrix, then in the paper [12] it is proved that the discrete analog of the maximum principle holds even without any convexity type assumptions. Further, if additionally by the matrix function $A(u, t)$ is independent of 
$u$ and $b(u, w, t)=b_{1}(u, t)+b_{2}(w, t)$, then it is shown in [29] that the discrete maximum principle is a necessary and sufficient condition for optimality.

\section{Discussions. Example}

The statement of theorem 4.1, in principle is a discrete analog of Pontryagin's maximum principle for the problem (2.1)-(2.3). If in the problem (2.1)-(2.3) we suppose $h=0$, then theorem 4.1 in a more enhanced form was proved in [22]. Additionally, using characteristic details of research methods of [21,22], we can strengthen the statement of theorem 4.1. We also note that if in problem (2.1)(2.3) we have $f(\cdot)=f\left(x(t), u(t), u\left(t-h_{0}\right), u\left(t-h_{1}\right), \ldots, u\left(t-h_{k}\right), t\right)$, where $h_{0}<h_{1}<\ldots<h_{k}=: h^{*}, h_{i} \in N$ and $t_{1}-t_{0}>h^{*}$, then in our technique, we should take we should take maximum delay $h^{*}$ instead of $h$.

In conclusion, we give an example showing the content-richness of theorem 4.1.

Example 5.1. Let us consider the problem

$$
\begin{gathered}
S(u(\cdot))=x_{2}(3) \rightarrow \min _{u(\cdot)}, \\
\left\{\begin{array}{c}
x_{1}(t+1)=(1-t) u(t-1) x_{1}(t)+(2-t) u(t), \\
x_{2}(t+1)=-x_{1}^{2}(t)+x_{2}(t)+u^{2}(t)+u^{2}(t-1), \\
x_{1}(0)=x_{2}(0)=0, t \in\{0,1,2\}=: I,
\end{array}\right. \\
u(t) \in U(t), t \in\{-1,0,1,2\}=: I_{h}, h=1, t_{1}=3,
\end{gathered}
$$

where $U(-1)=\{-1,0,1\}, U(0)=\{-1\} \cup[1,3], U(1)=U(2)=\{-1,0,1\}$. Study optimality of the admissible control $\bar{u}(t), t \in\{-1,0,1,2\}$, where $\bar{u}(-1)=$ $0, \bar{u}(0)=2, \bar{u}(1)=\bar{u}(2)=1$. By $(3.8)$ and $(3.11)$ we have $\bar{x}(0)=(0,0)^{T}, \bar{x}(1)=$ $(4,4)^{T}, \bar{x}(2)=(1,-7)^{T}, \bar{x}(3)=(-1,-6)$;

$$
\begin{gathered}
\bar{\psi}(0)=(8,-1)^{T}, \psi(1)=(2,-1)^{T}, \psi(2)=(0,-1)^{T} ; \\
f^{[0]}(0, \vartheta)=f(\bar{x}(0), \vartheta, \bar{u}(-1), 0)=\left(2 \vartheta, \vartheta^{2}\right)^{T}, \vartheta \in U(0), \\
f\left(f^{[0]}(0, \vartheta), \bar{u}(1), \vartheta, 1\right)=\left(1,1-2 \vartheta^{2}\right)^{T}, \vartheta \in U(0)=\{-1\} \cup[1,3] .
\end{gathered}
$$

Note that optimality conditions from $[14,29]$ cannot be applied because the sets $U(t), t \in\{-1,0,1,2\}$ are not convex and open and the sets $f(\bar{x}(t), U(t)$, $\bar{u}(t-h), t)$ and $f(\bar{x}(t), \bar{u}(t), U(t-h), t), t \in I$ are not convex, where $f(\cdot)$ and $U(\cdot)$ are determined by problem (5.1)- (5.3).

Apply theorem 4.1. It is easy to verify that optimality conditions (4.1) and (4.3) cannot be applied since the sets $f(\bar{x}(0), \bar{u}(0), U(-1), 0)$ and $f(\bar{x}(2), U(2)$, $\bar{u}(1), 2)$ do not satisfy assumptions of theorem 4.1. As the set $f\left(f^{[0]}(0, U(0))\right.$, $\bar{u}(1), U(0), 1)=\{1\} \times[-8,-1]$ is convex, then by statements $(4.2)$ of theorem 4.1 for $\theta=0$ we have:

$$
(-2,-1)\left[\left(1,1-2 \vartheta^{2}\right)^{T}-(1,-7)^{T}\right]=2\left(\vartheta^{2}-4\right) \leq 0, \forall \vartheta \in\{-1\} \cup[1,3] .
$$

Hence we get that by theorem 4.1, in the problem (5.1)-(5.3) the admissible control $\bar{u}(t), t \in\{-1,0,1,2\}$ is not optimal since the inequality (5.4) is not fulfilled. 


\section{References}

[1] L.T.Ashchepkov Optimal control with discontinuous systems Moscow, Nauka 1987 [in Russian].

[2] A.G.Butkovskii, On necessary and suocient optimality conditions for impulse control systems, Avtomat. I Telemekh. 24 (1963), no.8, 1056-1064.

[3] D.H.Chyung, Discrete optimal systems with time delay, IEEE Trans. Autim Control AC-13 (1) (1968).

[4] L-C Fan, W. Chu-Sen, Discrete maximum principle, Moscow, Mir 1967.

[5] R.Gabasov, F.M.Kirillova, Quality theory of optimal processes, Nauka 1971, 508.

[6] R. F. Gabasov and F. M. Kirillova, Necessary optimality conditions of equality type in discrete systems, Differ. Uravn. 9 (1973), no.3 542-546.

[7] A. Halanay, Optimal controls for systems with time lag, SIAM J.Control 6 (1968), no.2, 215-234.

[8] H. Halkin, On the necessary conditions for the optimal control of nonlinear systems, J. Math. Anal. 12 (1964), 1-12.

[9] G.L.Haratisvili and T.A.Tadumadze, Nonlinear optimal control systems with variable time lags, Math. USSR-Sb. 36 (1979), no. 6, 863-881.

[10] M.Yu. Kiseleva and V.I.Smagin, Control with a prognostic model with allowance for control delay, Vestn. Tomsk. Gos. Univ. Upr., Vychisl. Tekh. Inf. 11 (2010), no.2, $5-12$.

[11] S.T. Malik, On necessary optimality conditions for singular controls in dynamical systems with a delay in control, Numer. Funct.Anal. Optim. 39 (2018), no.15, 16691689.

[12] S.T.Malik, On the theory of necessary optimality conditions in discrete systems with a delay in control, Transactions of NAS of Azerbaijan, Issue Mathematics 38 (2018), no.1, 105-113

[13] K.B. Mansimov, On optimality of singular controls in the systems with delay controlled by means of initial functions, Dif.Uravn. 25 (1985), no.6, 1081-84.

[14] M.J. Mardanov and S.T. Malik, Necessary first-and second-order optimality conditions in discrete systems with a delay in control, J. Dyn. Control Syst. 25 (2019), no.1, 29-43.

[15] M.J. Mardanov, T.K. Melikov, S.T.Malik and K. Malikov, First-order and secondorder necessary conditions with respect to components for discrete optimal control problems, J. Comput. Appl. Math. 2020, 364.

[16] M.J.Mardanov, Legendre necessary conditions in delay control optimization problems, Dokl.Akad. Nauk SSSR 297 (1987), no.4, 795-797.

[17] M.J.Mardanov, Necessary optimality conditions in systems with lags and phase constraints, Math. Notes 42 (1987), no.5, 880-887.

[18] M.J. Mardanov and T.K.Melikov, A new discrete analogue of Pontryagin's maximum principle, Doklady Math. 98 (2018), no. 3, 549-551.

[19] M.J.Mardanov, T.K. Melikov, Analogue of the Kelley condition for optimal systems with retarded control, International Journal of Control 90 (2017), no. 7 1299-1307.

[20] M.J. Mardanov, T.K.Melikov, On necessary optimality conditions for discrete control systems, The Bulletin of Irkutsk State University. Series Mathematics 31 (2020), 49-61.

[21] M.J.Mardanov, T.K. Melikov and S.T. Malik, On strengthening of optimality conditions in discrete control systems, Proc. Inst. Math. Mech. 44 (2018), no.1, 135-154.

[22] M.J.Mardanov, T.K. Melikov, S.T. Malik, On the Theory of Optimal Processes in Discrete Systems, Mathematical Notes 106 (2019), no.(3-4) 390-401.

[23] A.S.Matveev, Optimal control problems with delays of general form and with phase constraints, Math. USSR-Izv. 33 (1989), no. 3, 521-552. 
[24] T.K.Melikov, Recurrent optimality conditions for singular controls in delay systems, Dokl. Akad. Nauk 322 (1992), no. 5, 843-846.

[25] T.K.Melikov, An analogue of Pontryagin's maximum principle in systems with neutral-type delay, Computational mathematics and mathematical physics $\mathbf{3 6}$ (1996), no.11, 1541-1546.

[26] B.S.Mordukhovich, Approximation methods in problems of optimization and control [in Russian]. Moscow: Nauka. 1988, 360.

[27] L.S.Pontryagin, V.G.Boltyanskii, R.V.Gamkrelidze, E.F. Mishchenko, Mathematical Theory of Optimal Processes, New York, Wiley 1962.

[28] A.I.Propoi, The maximum principle for discrete control systems, Autom. Remote Control 26 (1965), no.7, 1167-1177.

[29] F.K. Shak, The theory of optimal control by discrete processes, USSR Comput. Math. Math. Phys. 10 (1970), no.3, 68-84.

Misir J. Mardanov

Institute of Mathematics and Mechanics of NAS of Azerbaijan Baku, Azerbaijan, Baku State University, Baku, Azerbaijan

E-mail address: misirmardanov@yahoo.com

Samin T. Malik

Baku Higher Oil School, Baku, Azerbaijan

E-mail address: saminmelik@gmail.com

Received: June 12, 2020; Revised: October 16, 2020; Accepted: October 20, 2020 